\title{
STRICT RADICAL CLASSES OF ASSOCIATIVE RINGS
}

\author{
PATRICK N. STEWART
}

\begin{abstract}
A class of rings is strongly hereditary if it is closed under taking subrings. Strict radical classes (that is, radical classes for which the class of semisimple rings is strongly hereditary) are studied, strongly hereditary strict radical classes are classified, and it is shown that the category of associative rings has no proper localizing subcategories in the sense of Sul'geifer.
\end{abstract}

1. Introduction. In this paper we assume a familiarity with the basic notions of radical theory (see [3]). Let $\mathscr{R}$ be a radical class of associative rings and $\mathscr{S}$ the class of $\mathscr{R}$ semisimple rings. The radical class $\mathscr{R}$ is strict if $\mathscr{S}$ is closed under taking subrings; that is, if $\mathscr{S}$ is strongly hereditary. Notice that $\mathscr{R}$ is a strict radical class if and only if for each ring $A$, $\mathscr{R}(A)$ contains all subrings of $A$ which are in $\mathscr{R}$; or equivalently, if $S$ is a subring of $A$ and $S \in \mathscr{R}$, then the ideal of $A$ which is generated by $S$ is contained in $\mathscr{R}(A)$.

Kurosh [5] introduced the concept of a strict radical class for groups, and Livshits [6] and Sul'geifer [7] have studied strict radical classes in categories. More recently, Gardner [4] has proved that all $\mathscr{A}$-radical classes are strict (a radical class $\mathscr{R}$ of associative rings is an $\mathscr{A}$-radical class if whenever $A \in \mathscr{R}$ and $A^{+} \cong S^{+}$, then $S \in \mathscr{R}$; that is, if $\mathscr{R}$ depends only on the underlying additive group structure of rings), and Sul'gelfer [8] has employed the notion of a strongly hereditary strict radical class to develop a theory of localizing subcategories which applies to a wide class of nonabelian categories including the category of associative rings.

In this paper we investigate strict radical classes of associative rings and show that the category of associative rings has no proper localizing subcategories in the sense of Sul'gelfer.

2. Strict radical classes. Let $\mathscr{C}$ be a class of rings. Denote by $\mathscr{C}_{s}$ the class of all rings $A$ such that every nonzero homomorphic image of $A$ contains a nonzero subring which is (isomorphic to a ring) in $\mathscr{C}$. It follows from [3, Theorem 1] that $\mathscr{C}_{s}$ is a radical class.

Received by the editors October 16, 1972.

AMS (MOS) subject classifications (1970). Primary 16A21; Secondary 18E35.

Key words and phrases. Radical class, strict radical class, strongly hereditary radical class, localizing subcategory.

(c) American Mathematical Society 1973 
THEOREM 2.1. A radical class $\mathscr{R}$ is strict if and only if $\mathscr{R}=\mathscr{C}_{s}$ for some class of rings $\mathscr{C}$.

Proof. $^{1}$ It is clear that if $\mathscr{R}$ is a strict radical class, then $\mathscr{R}=\mathscr{R}_{s}$.

Let $\mathscr{C}$ be any class of rings, $A$ a ring and $S$ a subring of $A$. To establish that $\mathscr{C}_{s}$ is a strict radical class it is sufficient to prove that if $S \in \mathscr{C}_{s}$, then $S=S+A S+S A+A S A$ (the ideal of $A$ which is generated by $S$ ) is also in $\mathscr{C}_{s}$. Let $S / K$ be a nonzero homomorphic image of $S$. It is enough to find a nonzero homomorphism of $S$ into $S / K$.

If $S \nsubseteq K$, then the mapping defined by $x \rightarrow x+K$ for each $x \in S$ will suffice.

If $S \subseteq K$, then $S^{3} \subseteq K$ by Andrunakievič's lemma [3, Lemma 61]. Choose $n \geqq 2$ minimal such that $\bar{S}^{n} \subseteq K$. Then $\bar{S}^{n-1} \ddagger K$ so $(S+A S) \bar{S}^{n-2} \ddagger K$ (if $n=2$, take $S^{n-2}=A^{*}=$ the ring obtained by adjoining an identity to $A$ in the usual way). Now choose an integer $m$, an element $a \in A$, and an element $b \in \bar{S}^{n-2}$ such that $(m+a) S b \nsubseteq K$.

Consider the mapping defined by $x \rightarrow(m+a) x b+K$ for each $x \in S$. Clearly this mapping is well defined and behaves well with respect to addition. If $x_{1}, x_{2} \in S$, then

and

$$
(m+a) x_{1} x_{2} b \in A^{*} S^{2} S^{n-2} \subseteq \bar{S}^{n} \subseteq K
$$

$$
(m+a) x_{1} b(m+a) x_{2} b \in A^{*} S \bar{S}^{n-2} S \bar{S}^{n-2} \subseteq \bar{S}^{n} \subseteq K .
$$

Therefore the mapping is a homomorphism and since $(m+a) S b \neq K$, it is nonzero.

COROLlaRY 2.2. Let $\mathscr{C}$ be a class of rings and $A$ a ring.

(i) If $\mathscr{C}$ is homomorphically closed, then $A$ is $\mathscr{C}_{s}$ semisimple if and only if no nonzero subring of $A$ is in $\mathscr{C}$.

(ii) If $\mathscr{C}$ is a radical class, then $A$ is $\mathscr{C}_{s}$ semisimple if and only if every subring of $A$ is $\mathscr{C}$ semisimple.

COROLlaRy 2.3. A radical class $\mathscr{R}$ is strict if and only if $\mathscr{R}$ is the upper radical class determined by a strongly hereditary class of rings.

The proof of 2.3 follows from the observation that if $\mathscr{M}$ is a strongly hereditary class of rings, then the upper radical class determined by $\mathscr{M}$ is the radical class $\mathscr{C}_{s}$ where $\mathscr{C}$ is the class of all rings not in $\mathscr{M}$.

The generalized nil radical class $\mathscr{N}_{g}$ of Andrunakievič [1] and Thierrin [10] is the upper radical class determined by the class of all rings without proper divisors of zero. It follows from 2.3 that $\mathscr{N}_{g}$ is strict. In fact, it is

${ }^{1}$ This proof is patterned after the proof of Lemma 2 in Sulinski, Anderson and Divinsky, Lower radical properties for associative and alternative rings, J. London Math. Soc. 41 (1966), 417-424. MR 33 \#4095. 
known (see [2], [8]) that a ring is $\mathscr{N}_{g}$ semisimple if and only if it has no nonzero nilpotent elements, so it follows from 2.2(ii) that $\mathscr{N}_{g}=\mathscr{N}_{s}$ where $\mathscr{N}$ is the nil radical class.

3. Related rings. Let $\mathscr{R}$ be a strict radical class and $A$ an $\mathscr{R}$ semisimple ring. The ring $A_{n}$ of $n \times n$ matrices with entries from $A$ may not be $\mathscr{R}$ semisimple: the generalized nil radical class provides an example. Nor is the polynomial ring $A[x]$ necessarily $\mathscr{R}$ semisimple. To construct an example consider the polynomial ring over $Z_{2}$, the field of two elements. Notice that the ideal $I$ of $Z_{2}[x]$ which is generated by $x+x^{2}$ cannot be homomorphically mapped onto $Z_{2}$. Let $\mathscr{C}$ be the class of all homomorphic images of $I$. Then $Z_{2}$ is $\mathscr{C}_{s}$ semisimple but $\mathscr{C}_{s}\left(Z_{2}[x]\right)=I$. However, for any strict radical class $\mathscr{R}$, if $A$ is $\mathscr{R}$ semisimple, then $\mathscr{R}(A[x]) \subseteq$ $\left\{f \in A[x]: f(a)=0\right.$ for each $a$ in the centre of $\left.A^{*}\right\}$. This follows because there can be no nonzero homomorphisms of a ring in $\mathscr{R}$ into an $\mathscr{R}$ semisimple ring.

Although the above examples show that in general $\mathscr{R}\left(A_{n}\right) \neq \mathscr{R}(A)_{n}$ and $\mathscr{R}(A[x]) \neq \mathscr{R}(A)[x]$ for a strict radical class $\mathscr{R}$, we do have the following result.

Proposition 3.1. For any strict radical class $\mathscr{R}$,

(i) if $A \in \mathscr{R}$, then $A_{n} \in \mathscr{R}$,

(ii) if $A \in \mathscr{R}$, then $A[x] \in \mathscr{R}$.

Proof. (i) Let $\mathscr{R}$ be a strict radical class and suppose that $A \in \mathscr{R}$. Then $A$ (more precisely, the subring of scalar matrices) is contained in $\mathscr{R}\left(A_{n}\right)$ and so $\mathscr{R}\left(A_{n}\right) \supseteq A\left(A_{n}\right)=\left(A_{n}\right)^{2}$. Since $A_{n} /\left(A_{n}\right)^{2}$ is isomorphic to the direct sum of $n^{2}$ copies of $A / A^{2}, A_{n} /\left(A_{n}\right)^{2} \in \mathscr{R}$. Therefore, $A_{n} \in \mathscr{R}$.

The proof of (ii) is similar.

4. Sul'geifer's localizing subcategories. Using the notion of a strongly hereditary strict radical class Sul'geifer [8] has developed a theory of localizing subcategories which is applicable to the category of associative rings. However, in this section we shall prove that the category of associative rings has no proper localizing subcategories in the sense of Sul'gerfer.

For each set $S$ of prime numbers let $T_{S}$ denote the class of all rings $A$ such that for each $x \in A, p_{1}^{\alpha_{1}} \cdots p_{k}^{\alpha_{k}} x=0$ for some $p_{i} \in S$ and positive integers $\alpha_{1}, \cdots, \alpha_{k}$ depending on $x$. When $S$ is the set of all prime numbers we shall denote $T_{S}$ by $T$, and we set $T_{\phi}=\{(0)\}$. It is clear that each $T_{S}$ is a strongly hereditary strict radical class. In the next proposition we show that, except for the class of all rings, these are the only strongly hereditary strict radical classes. 
Proposition 4.1. A strict radical class $\mathscr{R}$ which is not the class of all rings is strongly hereditary if and only if $\mathscr{R}=T_{S}$ for some set $S$ of prime numbers.

PRoof. Assume that $\mathscr{R}$ is a strongly hereditary strict radical class. First we show that if $\mathscr{R} \nsubseteq T$, then $\mathscr{R}$ is the class of all rings.

Suppose that $(0) \neq A \in \mathscr{R}$ and that $A$ is $T$ semisimple. By 3.1(i) the matrix ring $A_{2} \in \mathscr{R}$ and so $C^{\infty}$, the zero ring on the infinite cyclic group, is in $\mathscr{R}$ because it is isomorphic to a subring of $A_{2}$. Thus the simple ring $Q_{2}$, where $Q$ is the field of rational numbers, is in $\mathscr{R}$ since it has a subring isomorphic to $C^{\infty}$. But then all subrings of $Q_{2}$ are in $\mathscr{R}$ and so $Z$, the ring of integers, is in $\mathscr{R}$. Now, by 3.1(ii), $Z[x] \in \mathscr{R}$ from which it follows that all rings which are generated by one element are in $\mathscr{R}$. Therefore $\mathscr{R}$ is the class of all rings.

If $\mathscr{R}$ is a strongly hereditary strict radical class which is not the class of all rings, then $\mathscr{R} \subseteq T$. Arguing as above we see that if $p$ is a prime number such that $\mathscr{R} \cap T_{\{p\}} \neq\{(0)\}$, then $T_{\{p\}} \subseteq \mathscr{R}$. It follows that $\mathscr{R}=T_{S}$ where $S=\left\{p: \mathscr{R} \cap T_{\{p\}} \neq\{(0)\}\right\}$. This completes the proof of the proposition.

Let $\mathscr{R}$ be a strongly hereditary strict radical class. A ring $C$ is $\mathscr{R}$ closed $[8,4.16]$ if $C$ is $\mathscr{R}$ semisimple and if each homomorphism $A \rightarrow C$ can be extended to a homomorphism $B \rightarrow C$ whenever $A$ is an $\mathscr{R}$ accessible subring of $B$. The general definition of $\mathscr{R}$ accessible is given in [8, 4.13]; for our purposes the following characterization $[8,4.14]$ is sufficient: a subring $A$ of an $\mathscr{R}$ semisimple ring $B$ is an $\mathscr{R}$ accessible subring of $B$ if and only if there is a finite chain of subrings $I_{1} \subseteq I_{2} \subseteq \cdots \subseteq I_{k}=B$ such that $I_{1} \subseteq A$ and $I_{i}$ is an ideal of $I_{i+1}$ with $I_{i+1} / I_{i} \in \mathscr{R}$ for each $i=1, \cdots, k-1$.

It follows from Sul'geifer [8, 4.12 and 4.19] that a subcategory of the category of associative rings is a localizing subcategory if and only if it is a full subcategory, the class of objects in the subcategory is a strongly hereditary strict radical class $\mathscr{R}$, and each $\mathscr{R}$ semisimple ring can be embedded as an $\mathscr{R}$ accessible subring in an $\mathscr{R}$ closed ring.

From 4.1 we see that the class of objects of a proper localizing subcategory must be $T_{S}$ for some nonempty set $S$ of prime numbers.

In the next example we exhibit a $T$ semisimple ring which can not be embedded as a $T$ accessible subring in a $T$ closed ring.

EXAMPLE 4.2. Let $Q\left[x_{1}, \cdots, x_{n}, \cdots\right]$ be the ring of polynomials in an infinite number of commuting indeterminates over the field of rational numbers $Q$. Let $D$ be the ideal of all polynomials with zero constant term and let $S$ be the subring of $D$ consisting of all polynomials with zero constant term and integer coefficients. Set

$$
A=S+\sum_{n=1}^{\infty} x_{n} D^{n} .
$$


Suppose that $C$ is a $T$ closed ring with subrings $I_{1}, \cdots, I_{k}$ such that

$$
I_{1} \subseteq I_{2} \subseteq \cdots \subseteq I_{k}=C, \quad I_{1} \subseteq A \subseteq C,
$$

and $I_{i}$ is an ideal of $I_{i+1}$ with $I_{i+1} / I_{i} \in T$ for each $i=1, \cdots, k-1$.

Choose $N>k$ and define

$$
A_{1}=A, \quad A_{i+1}=A_{i}+\sum_{n=i+1}^{\infty} x_{n} D^{n-i} \text { for } i=1, \cdots, N-1 .
$$

Since $A_{N}$ is $T$ semisimple and $A_{i}$ is an ideal of $A_{i+1}$ with $A_{i+1} / A_{i} \in T$ for each $i=1, \cdots, N-1, A$ is a $T$ accessible subring of $A_{N}$. Because $A$ is a subring of $C$ and $C$ is $T$ closed there is a homomorphism $f: A_{N} \rightarrow C$ which is the identity on $A$.

Each of the factor rings $I_{i+1} / I_{i}$ is in $T$ and $x_{N} \in A \subseteq C$, so there is an integer $m \geqq 2$ such that $m x_{N} \in I_{1}$. Since $x_{N} D \subseteq A_{N}, x_{N}^{2} / m^{k} \in A_{N}$. Now $f\left(m x_{N}\right)=m x_{N} \in I_{1}, f\left(x_{N}^{2} / m^{k}\right) \in C$, and $I_{i}$ is an ideal of $I_{i+1}$ for each $i=1, \cdots, k-1$, so

$$
f\left(x_{N}^{k+1} / m\right)=\left(f\left(m x_{N}\right)\right)^{k-1}\left(f\left(x_{N}^{2} / m^{k}\right)\right) \in I_{1} \subseteq A .
$$

Thus there is an element $a \in A$ such that

$$
m a=m f\left(x_{N}^{k+1} / m\right)=f\left(x_{N}^{k+1}\right)=x_{N}^{k+1} .
$$

However, from the definition of $A$ it is clear that this implies that $k+1 \geqq$ $N+1$ which contradicts the choice of $N$.

Therefore $A$ is not a $T$ accessible subring of any $T$ closed ring.

It is clear how to modify the above example so as to obtain an example for the other nontrivial strongly hereditary strict radical classes $T_{S}$. Thus we obtain the following.

THEOREM 4.3. The category of associative rings has no proper localizing subcategories in the sense of $\check{S} u l^{\prime}$ gěfer.

\section{REFERENCES}

1. V. Andrunakievič, Radicals in associative rings. II, Mat. Sb. 55 (97) (1961), 329-346; English transl., Amer. Math. Soc. Transl. (2) 52 (1966), 129-149. MR 25 \#1186.

2. V. Andrunakievič and Ju. M. Rjabuhin, Rings without nilpotent elements, and completely prime ideals, Dokl. Akad. Nauk SSSR 180 (1968), 9-11=Soviet Math. Dokl. 9 (1968), 565-568. MR 37 \#6320.

3. N. J. Divinsky, Rings and radicals, Univ. of Toronto Press, Toronto, 1965. MR 33 \#5654.

4. B. J. Gardner, Radicals of abelian groups and associative rings, Acta. Math. Acad. Sci. Hungar. (to appear).

5. A. G. Kuroš, Radicals in the theory of groups, Sibirsk. Mat. Ž. 3 (1962), 912-931. (Russian) MR 26 \#1363. 
6. A. H. Livšic, Category-theoretical foundations of the duality of radicality and semisimplicity, Sibirsk. Mat. Ž. 5 (1964), 319-336; English transl., Amer. Math. Soc. Transl. (2) 58 (1966), 57-76. MR 29 \#2288.

7. E. G. Sul'geifer, Functor characterization of strict radicals in categories, Sibirsk. Mat. Z. 7 (1966), 1412-1421 =Siberian Math. J. 7 (1966), 1105-1111. MR 34 \#4333.

8. - Localizations and strongly hereditary strict radicals in categories, Trudy Moskov. Mat. Obšč. 19 (1968), 271-301=Trans. Moscow Math. Soc. 1968, 299-331. MR 38 \#5881.

9. P. N. Stewart, Semi-simple radical classes, Pacific J. Math. 32 (1970), 249-254. MR 41 \#254.

10. G. Thierrin, Sur les ideaux complement premiers d'un anneaux quelconque, Bull. Acad. Roy. Belg. (5) 43 (1957), 124-132. MR 19, 383.

Department of Mathematics, The University of Adelame, Adelaide, South Australia

Current address: Department of Mathematics, Dalhousie University, Halifax, Nova Scotia, Canada 